\title{
Auch Männer von Brustkrebs betroffen
}

\begin{abstract}
Die Diagnose Brustkrebs können auch Männer erhalten, wenngleich deutlich seltener als Frauen. Doch die Situation ist für betroffene Männer oft besonders schwierig. Welche speziellen medizinischen und psychosozialen Bedürfnisse männliche Brustkrebspatienten haben, wollen Wissenschaftler aus Bonn nun untersuchen. Die Deutsche Krebshilfe unterstützt das Projekt mit rund 155.000 Euro.
\end{abstract}

\begin{abstract}
rustkrebs ist mit etwa 65.500 Neuerkrankungen pro Jahr die häufigste Tumorerkrankung bei Frauen. Weitaus weniger bekannt ist: Auch Männer können betroffen sein. Nach Hochrechnungen des Robert Koch-Instituts Berlin erhalten rund 700 Männer jedes Jahr die Diagnose Brustkrebs. Für die Betroffenen ist dies oft besonders belastend. Denn sie müssen die Krebsdiagnose verarbeiten und sehen sich zusätzlich mit dem Gefühl konfrontiert, an einer potenziellen „Frauenkrankheit“ zu leiden. In der Bevölkerung ist zudem wenig be-
\end{abstract}

kannt, dass auch Männer an Brustkrebs erkranken können, was zu einem Gefühl der Stigmatisierung und Ausgrenzung führen kann. Nicht nur für die Patienten, auch für die behandelnden Ärzte und Pflegekräfte ist die Situation oft sehr schwierig und ungewohnt.

Welche speziellen Bedürfnisse haben männliche Brustkrebspatienten? Ist die medizinische Versorgung, die für Frauen stetig verbessert wurde, auch für Männer die bestmögliche? Diese und weitere Fragen wollen Wissenschaftler des Universitätsklinikums Bonn nun be-

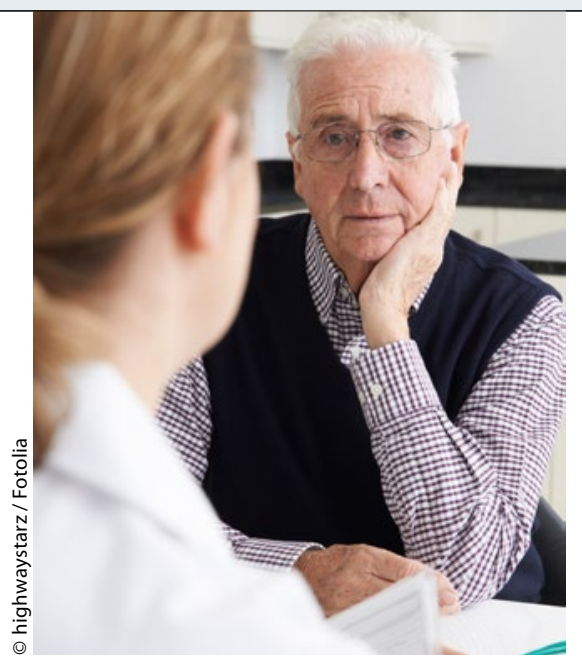

antworten. Gemeinsam mit der Klinik und Poliklinik für Psychosomatik und Psychotherapie der Universität Köln, dem Brustzentrum des Klinikums der Universität München, der Deutschen Krebsgesellschaft und dem Netzwerk Männer mit Brustkrebs e.V. erfassen sie die medizinischen und psychosozialen Bedürfnisse von männlichen Brustkrebspatienten und werten diese aus.

www.krebshilfe.de

\section{Hier steht eine Anzeige.}

\section{Springer}

